\title{
Correlation of Intraoperative End-Tidal Carbon Dioxide Concentration on Postoperative Hospital Stay in Patients Undergoing Pylorus-Preserving Pancreaticoduodenectomy
}

\author{
Ji-Hoon Park ${ }^{1} \cdot$ Hye-Mi Lee ${ }^{2,3}$ - Chang Moo Kang ${ }^{4,5} \cdot$ Kyung Sub Kim ${ }^{6}$ Chul Ho Jang ${ }^{6}$ \\ Ho Kyoung Hwang ${ }^{4,5} \cdot$ Jeong-Rim Lee $\mathrm{L}^{2,3}$
}

Accepted: 24 January 2021/Published online: 16 February 2021

(C) Société Internationale de Chirurgie 2021

\begin{abstract}
Background Hypocapnia has been traditionally advocated during general anesthesia, even though it may induce deleterious physiological effects that result in unfavorable outcomes in patients. This study investigated the association between intraoperative end-tidal carbon dioxide $\left(\mathrm{EtCO}_{2}\right)$ and length of hospital stay (LOS) in patients who underwent pylorus-preserving pancreaticoduodenectomy (PPPD).

Methods The medical records of 759 patients from 2006 to 2015 were reviewed. The patients were divided into two groups based on the mean $\mathrm{EtCO}_{2}$ value during general anesthesia: the hypocapnia group $(<35 \mathrm{mmHg})$ and the normocapnia group $(\geq 35 \mathrm{mmHg}$ ). The primary outcome was LOS between the groups. Secondary outcomes included the length of intensive care unit (ICU) stay, postoperative 30-day, 1-year, and 2-year mortality, and perioperative factors associated with LOS.

Results A total of 727 patients were finally analyzed. The median LOS of the hypocapnia group was significantly longer than that of the normocapnia group (22 days vs. 18 days, respectively; $p<0.001$ ). Postoperative mortality did not differ between the groups. Cox regression analysis revealed that hypocapnia was an independent risk factor for longer LOS (hazard ratio [HR], 1.61; 95\% confidence interval [CI], 1.37-1.89; $p<0.001$ ). Age and postoperative pancreatic fistula were also risk factors for a longer LOS.

Conclusions It was concluded that low levels of intraoperative $\mathrm{EtCO}_{2}$ during general anesthesia were associated with an increased LOS for patients undergoing PPPD.
\end{abstract}

Ji-Hoon Park and Hye-Mi Lee: Co-first author.

Ho Kyoung Hwang

drhhk@yuhs.ac

$\triangle$ Jeong-Rim Lee leejeongrim@gmail.com

1 Department of Anesthesiology and Pain Medicine, Keimyung University Dongsan Medical Center, 1095 Dalgubeol-daero, Dalseo-gu, Daegu 42601, Korea

2 Department of Anaesthesiology and Pain Medicine, Yonsei University College of Medicine, 50-1 Yonsei-ro, Seodaemungu, Seoul 03722, Republic of Korea

3 Anesthesia and Pain Research Institute, Yonsei University College of Medicine, 50-1 Yonsei-ro, Seodaemun-gu, Seoul 03722, Korea
4 Division of Hepatobiliary and Pancreatic Surgery, Department of Surgery, Yonsei University College of Medicine, 50-1 Yonsei-ro, Seodaemun-gu, Seoul 03722, Republic of Korea

5 Yonsei Cancer Center, Pancreatobiliary Cancer Clinic, Severance Hospital, 50-1 Yonsei-ro, Seodaemun-gu, Seoul 03722, Korea

6 Department of Anesthesiology and Pain Medicine, Yonsei University College of Medicine, Gangnam Severance Hospital, 211 Eonju-ro, Gangnam-gu, Seoul 06273, Korea 


\section{Introduction}

Pylorus-preserving pancreaticoduodenectomy (PPPD) is one of the most invasive intraabdominal surgeries; it requires a prolonged recovery time after surgery because of its extensive incision, invasiveness, severe postoperative pain, decreased lung function, and postoperative complications [1]. Therefore, it is important to seek out every available opportunity to speed up recovery in order for patients to return to their daily lives as soon as possible after the operation.

Hypocapnia caused by hyperventilation during general anesthesia is a common situation. Traditionally, it has been traditionally advocated, to some extent, because it has been considered to prevent the restoration of spontaneous respiratory efforts and, thus, may reduce the need for muscle relaxants and additional anesthetics [2]. However, hypocapnia has several possible disadvantages in terms of maintaining a normal physiology as it can reduce cerebral blood flow and oxygen delivery [3]. Hypocapnia also causes an increase in the capillary permeability of the lungs, bronchoconstriction, an increase in shunt as a result of decreased lung compliance, and a leftward shift of the hemoglobin dissociative curve [4]. Moreover, it reduces splanchnic blood flow and increases myocardial oxygen demand, hypercoagulability, and the incidence of dysrhythmias [4]. Although the arterial partial pressure of carbon dioxide $\left(\mathrm{PaCO}_{2}\right)$ is not monitored continuously, its status is well reflected by endtidal carbon dioxide $\left(\mathrm{EtCO}_{2}\right)$ levels, which are routinely monitored during anesthesia [5]. Despite these shortcomings, $\mathrm{EtCO}_{2}$ tends to remain as low as ever during surgery [2], and poor clinical outcomes related to hypocapnia have not been sufficiently studied [6, 7].

Prior studies have shown that intraoperative hypocapnia is related to worse outcomes in patients undergoing lower abdominal surgery $[6,7]$. However, it is unknown whether deleterious effects from hypocapnia exist in other surgeries, such as PPPD. We hypothesized that hypocapnia in patients undergoing anesthesia for PPPD is associated with worse clinical outcomes compared with patients maintained under normocapnia or hypercapnia.

In this retrospective study, we investigated the effect of intraoperative hypocapnia on hospital stay and mortality in patients undergoing PPPD.

\section{Materials and methods}

\section{Ethics}

This study was approved by the institutional review board (IRB) of Severance Hospital (no. 4-2019-1164), and the need for informed consent was waived.

\section{Subjects}

Data were retrospectively collected from patients who underwent PPPD for a suspicious malignancy at a tertiary university hospital (Severance Hospital) from January 2006 to December 2015. Patients were excluded if they expired during surgery, underwent surgery assisted by laparoscopy or robot, or had undergone cooperation or reoperation.

The patients were divided into two groups based on their mean $\mathrm{EtCO}_{2}$ values during anesthesia, namely the hypocapnia group (less than $35 \mathrm{mmHg}$ ) and the normocapnia group (equal to or greater than $35 \mathrm{mmHg}$ ), as proposed by Way and Hill [8].

\section{Perioperative variables}

We collected data related to subject age, sex, height, weight, body mass index (BMI), comorbidities, American Society of Anesthesiologists (ASA) classification, tumor location, and malignancy. The collected intraoperative variables were the duration of operation, duration of anesthesia, and peak inspiratory airway pressure (PIP), and $\mathrm{EtCO}_{2}$, which was recorded every $15 \mathrm{~min}$ in the anesthesia records. Hemodynamic data including heart rate, systolic blood pressure (SBP), diastolic blood pressure (DBP), and central venous pressure (CVP) were recorded every $5 \mathrm{~min}$ during surgery. Laboratory data, including $\mathrm{PaCO}_{2}$ from arterial blood gas analysis (ABGA), were also recorded every hour during the operation. The mean values of the variables collected during operation were used for analysis.

\section{Outcomes}

The primary outcome was the comparison of postoperative length of hospital stay (LOS) after surgery between the groups. Secondary outcomes included the length of ICU stay; postoperative 30-day, 1-year and 2-year mortality; and incidence of postoperative pancreatic fistula (POPF) as the major postoperative complication [9].

\section{Statistical analysis}

Statistical analyses were performed using IBM SPSS $^{\circledR}$ Statistics software (ver. 24.0; IBM Corp., Armonk, NY, USA) and SAS (version 9.4, SAS Inc., Cary, NC, USA). For the assumption of a normal distribution, we used the Shapiro-Wilk tests. According to the normality of the data, continuous variables (age, height, weight, body mass index (BMI), duration of operation, duration of anesthesia, intraoperative vital signs, $\mathrm{EtCO}_{2}$, and $\mathrm{PaCO}_{2}$ ) were 
analyzed using Student's t test or Mann-Whitney U test and were reported as the mean \pm standard deviation (SD) or median [interquartile range (IQR)]. All categorical and ranking variables (sex, ASA classification, tumor location, malignancy, mortality, and incidence of POPF) were analyzed using the $\chi^{2}$ test or Fisher's exact test and were expressed as $\mathrm{n}(\%)$.

Differences in LOS and ICU stay (in days) were calculated using the Kaplan-Meier analysis and log-rank test. Perioperative factors of LOS were analyzed using stepwise Cox regression analysis. Estimates for hazard ratios (HRs) and $95 \%$ confidence intervals (CIs) were calculated from linear regression models. Independent risk factors for LOS were studied using linear regression analysis. Eighteen variables relating to baseline status were selected for analysis. They included age, sex, operation time, ASA classification, intraoperative vital signs, comorbidities, POPF, and hypocapnia. The variables were tested for selection through stepwise variable selection and were assessed for their impact on LOS. A correlation analysis was conducted to determine the relative influence of $\mathrm{EtCO}_{2}$ and LOS. A value of $p<0.05$ was considered to indicate a significant difference for all analyses.
Results

A total of 756 patients were identified, and their medical histories were reviewed. A total of 727 patients were finally included (Fig. 1). Baseline characteristics of the patients are presented in Table 1. The mean $\mathrm{EtCO}_{2}$ of the hypocapnia group was $33.3 \pm 1.9 \mathrm{mmHg}$ versus $36.5 \pm 1.2 \mathrm{mmHg}$ in the normocapnia group $(p<0.001)$. The mean $\mathrm{PaCO}_{2}$ in the hypocapnia group was lower than that in the normocapnia group $(32.1 \pm 2.6$ vs. $33.4 \pm 2.4$, respectively; $p<0.001)$. Although the body weight in the normocapnia group was greater than that in the hypocapnia group, the BMI was comparable between the groups. The values of SBP, CVP, and PIP were statistically higher in the normocapnia group. There were more patients with ASA classification 3 in the normocapnia group than in the hypocapnia group.

\section{Primary outcome parameters}

The patients in the hypocapnia group had a significantly longer hospital stay than those in the normocapnia group (median [IQR], 22 days [16-29] vs. 18 days [13-24];

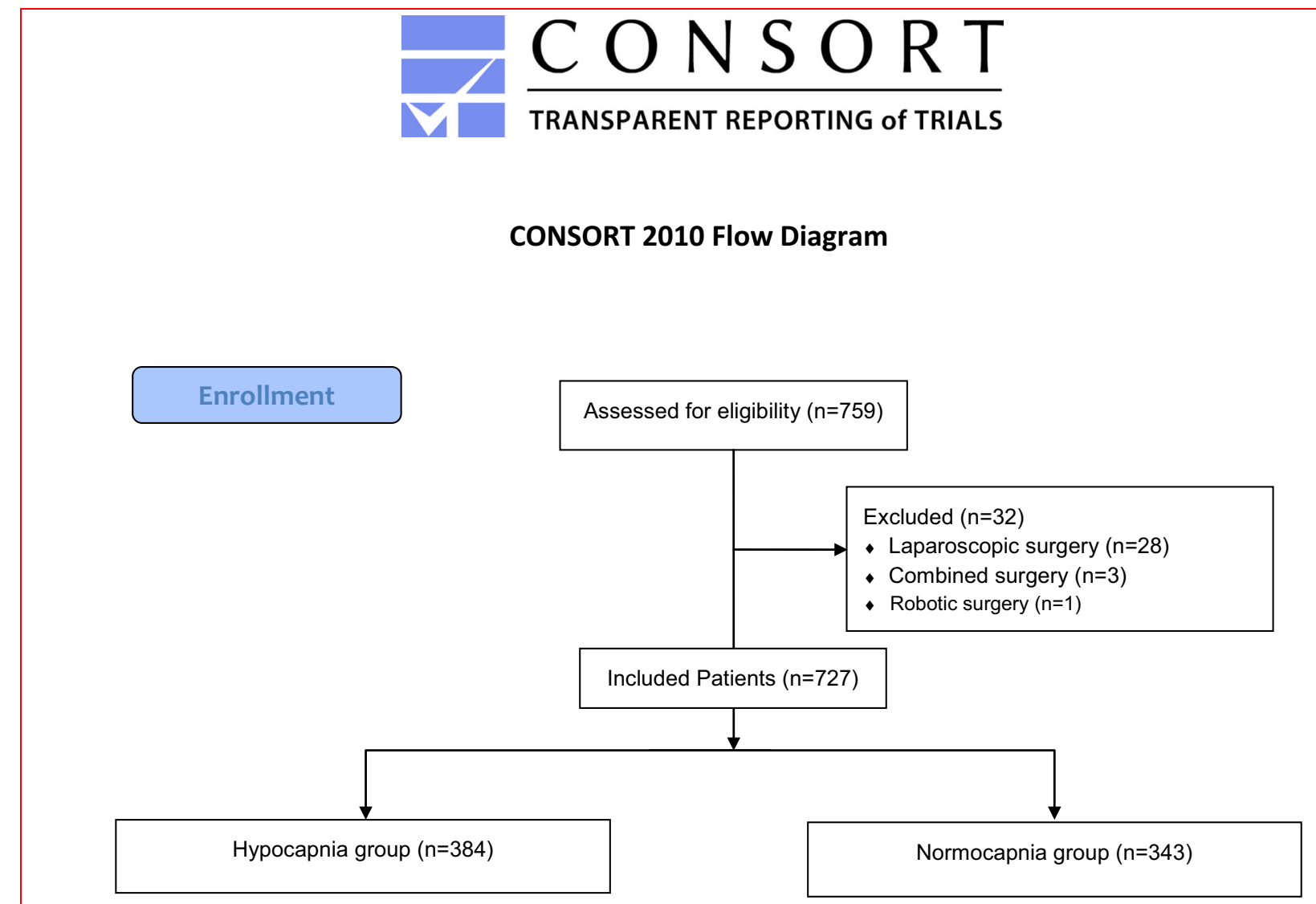

Fig. 1 CONSORT flow diagram of the study 
Table 1 Baseline characteristics of the patients included in the two study groups

\begin{tabular}{|c|c|c|c|}
\hline & Hypocapnia $(n=384)$ & Normocapnia $(n=343)$ & $p$ value \\
\hline Patient enrollment (year) & & & $<0.001$ \\
\hline 2006-2007 & $76(19.8)$ & $4(1.2)$ & \\
\hline 2008-2009 & $87(22.7)$ & $25(7.3)$ & \\
\hline 2010-2011 & $93(24.2)$ & $60(17.5)$ & \\
\hline 2012-2013 & $86(22.4)$ & $100(29.1)$ & \\
\hline 2014-2015 & $42(10.9)$ & $154(44.9)$ & \\
\hline Age (years) & $64[55-70]$ & $64[56-70]$ & 0.838 \\
\hline Height $(\mathrm{cm})$ & $162.0[156.0-168.0]$ & $164.0[156.9-170.0]$ & 0.115 \\
\hline Weight $(\mathrm{kg})$ & $60.0[53.5-67.9)$ & $63.0[55.4-68.3]$ & 0.025 \\
\hline Body mass index $\left(\mathrm{kg} \mathrm{m}^{-2}\right)$ & $22.9[21.3-24.8]$ & $23.3[21.5-25.4)$ & 0.108 \\
\hline Sex & & & 0.072 \\
\hline Male & $212(55)$ & $212(62)$ & \\
\hline Female & $172(45)$ & $131(38)$ & \\
\hline Tumor location & & & 0.796 \\
\hline Pancreas & $161(42)$ & $133(39)$ & \\
\hline Common bile duct & $105(27)$ & $109(32)$ & \\
\hline Ampulla of Vater & $88(23)$ & $83(24)$ & \\
\hline Duodenum & $23(6)$ & $16(5)$ & \\
\hline Gallbladder & $7(2)$ & $2(1)$ & \\
\hline Malignancy & $340(86)$ & $301(88)$ & 0.462 \\
\hline ASA classification & & & $<0.001$ \\
\hline 1 & $38(10)$ & $23(7)$ & \\
\hline 2 & $297(77)$ & $225(66)$ & \\
\hline 3 & $47(12)$ & $92(27)$ & \\
\hline 4 & $2(1)$ & $3(1)$ & \\
\hline Heart rate $\left(\min ^{-1}\right)$ & $73.7 \pm 10.2$ & $74.9 \pm 10.2$ & 0.099 \\
\hline Systolic blood pressure $(\mathrm{mmHg})$ & $113.4 \pm 7.4$ & $115.4 \pm 8.2$ & 0.037 \\
\hline Diastolic blood pressure $(\mathrm{mmHg})$ & $60.7 \pm 5.9$ & $60.7 \pm 6.2$ & 0.981 \\
\hline Central venous pressure $(\mathrm{mmHg})$ & $6.9 \pm 2.3$ & $7.4 \pm 2.5$ & $<0.001$ \\
\hline Peak airway pressure $\left(\mathrm{cmH}_{2} \mathrm{O}\right)$ & $14.3 \pm 2.7$ & $16.1 \pm 2.9$ & $<0.001$ \\
\hline Operation time (min) & $419 \pm 135$ & $422 \pm 104$ & 0.415 \\
\hline $\mathrm{EtCO}_{2}(\mathrm{mmHg})$ & $33.3 \pm 1.9$ & $36.5 \pm 1.2$ & $<0.001$ \\
\hline $\mathrm{PaCO}_{2}(\mathrm{mmHg})$ & $32.1 \pm 2.6$ & $33.4 \pm 2.4$ & $<0.001$ \\
\hline
\end{tabular}

Values are presented as the median [IQR] value or the number (\%) of patients or mean \pm standard deviation. ASA American Society of Anesthesiologists; $\mathrm{EtCO}_{2}$ end-tidal carbon dioxide; $\mathrm{PaCO}_{2}$ arterial partial pressure of carbon dioxide

$p<0.001$; Fig. 2 and Table 2). The correlation between $\mathrm{EtCO}_{2}$ and LOS showed a negative correlation with $r=-0.249, p<0.001$ (Fig. 3).

\section{Secondary outcome parameters}

The length of ICU stay and postoperative 30-day, 1-year, and 2-year mortality did not differ between the groups (Table 2). Cox multivariable analysis revealed that hypocapnia was an independent risk factor for longer LOS (HR, 1.61; 95\% CI, 1.37-1.89; $p<0.001$; Table 3). POPF was also an independent risk factor for LOS (HR, 1.41; 95\% CI, 1.18-1.69; $p<0.001$ ), but the incidence of POPF did not differ between the two groups (Tables 2, 3). Age and surgeon's experience were also significantly associated with a longer LOS. 
Fig. 2 Kaplan-Meier analysis of the effect of end-tidal carbon dioxide $\left(\mathrm{EtCO}_{2}\right)$ on length of hospital stay (LOS)

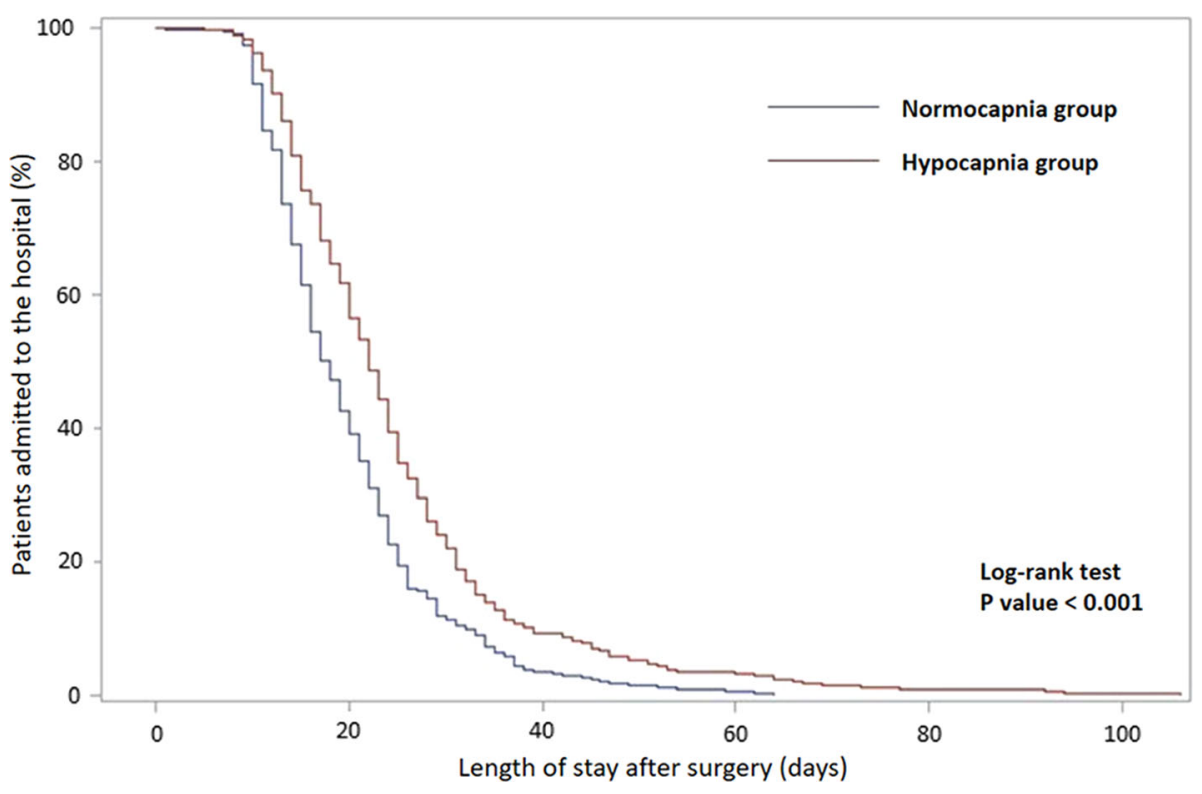

Table 2 Postoperative outcomes after pylorus-preserving pancreatoduodenectomy

\begin{tabular}{|c|c|c|c|c|}
\hline & $\begin{array}{l}\text { Hypocapnia } \\
(n=384)\end{array}$ & $\begin{array}{l}\text { Normocapnia } \\
(n=343)\end{array}$ & OR (95\% CI) & $p$ value \\
\hline LOS (day) & $22(16-29)$ & $18(13-24)$ & & $<0.001$ \\
\hline Length of ICU stays & $2(1-2)$ & $1(1-2)$ & & 0.13 \\
\hline 30-day mortality & $6(0.8)$ & $2(0.6)$ & $2.71(0.54-13.50)$ & 0.225 \\
\hline 1-year mortality & $47(12.2)$ & $32(9.3)$ & $1.36(0.84-2.18)$ & 0.209 \\
\hline 2-year mortality & $101(26.3)$ & $79(23.0)$ & $1.19(0.85-1.67)$ & 0.308 \\
\hline POPF & $87(22.7)$ & $92(26.8)$ & $1.25(0.89-1.75)$ & 0.193 \\
\hline Vascular resection & $5(1.3)$ & $14(4.1)$ & $0.31(0.11-0.87)$ & 0.019 \\
\hline
\end{tabular}

Values are presented as the median [IQR] or the number (\%) of patients. $C I$ confidence interval; $L O S$ length of hospital stay; $I C U$ intensive care unit; $P O P F$ postoperative pancreatic fistula

\section{Discussion}

This study shows that patients whose $\mathrm{EtCO}_{2}$ was less than $35 \mathrm{mmHg}$ during surgery had a longer hospital stay after PPPD, and this mild hypocapnia is an independent risk factor associated with a longer hospital stay (HR 1.61) when compared with patients with $\mathrm{EtCO}_{2} \geq 35 \mathrm{mmHg}$.

The length of hospital stay is a valid factor related to quality of care and patients' postoperative functioning [10]. Similar to the results of two other studies $[6,7]$, our investigation revealed that even mild hypocapnia can result in longer hospital stays and unwanted adverse outcomes. Because all three studies, including ours, were conducted retrospectively, we can surmise that unfavorable physiologic changes due to a low $\mathrm{EtCO}_{2}$ could result in detrimental outcomes. As mentioned earlier, hypocapnia has various effects on the whole body, most of which are negative. Hypocapnia, in particular, decreases tissue perfusion, reduces splanchnic blood flow, and affects pulmonary stress, all of which are likely related to the most common complications and factors associated with recovery. A couple of studies investigated the clinically significant effects of $\mathrm{EtCO}_{2}$ prospectively and demonstrated the harmful effects of hypocapnia in terms of postoperative nausea/vomiting or postoperative delirium [11, 12]. However, it is difficult to prove which aspects of the deteriorating pathophysiology directly affect particular organs or systems.

Meanwhile, mild hypercapnia is considered more beneficial after surgery [6, 13-15], while the mean $\mathrm{EtCO}_{2}$ in our normocapnic group was just $36.2 \mathrm{mmHg}$, and no patient was categorized as having hypercapnia. Our results 


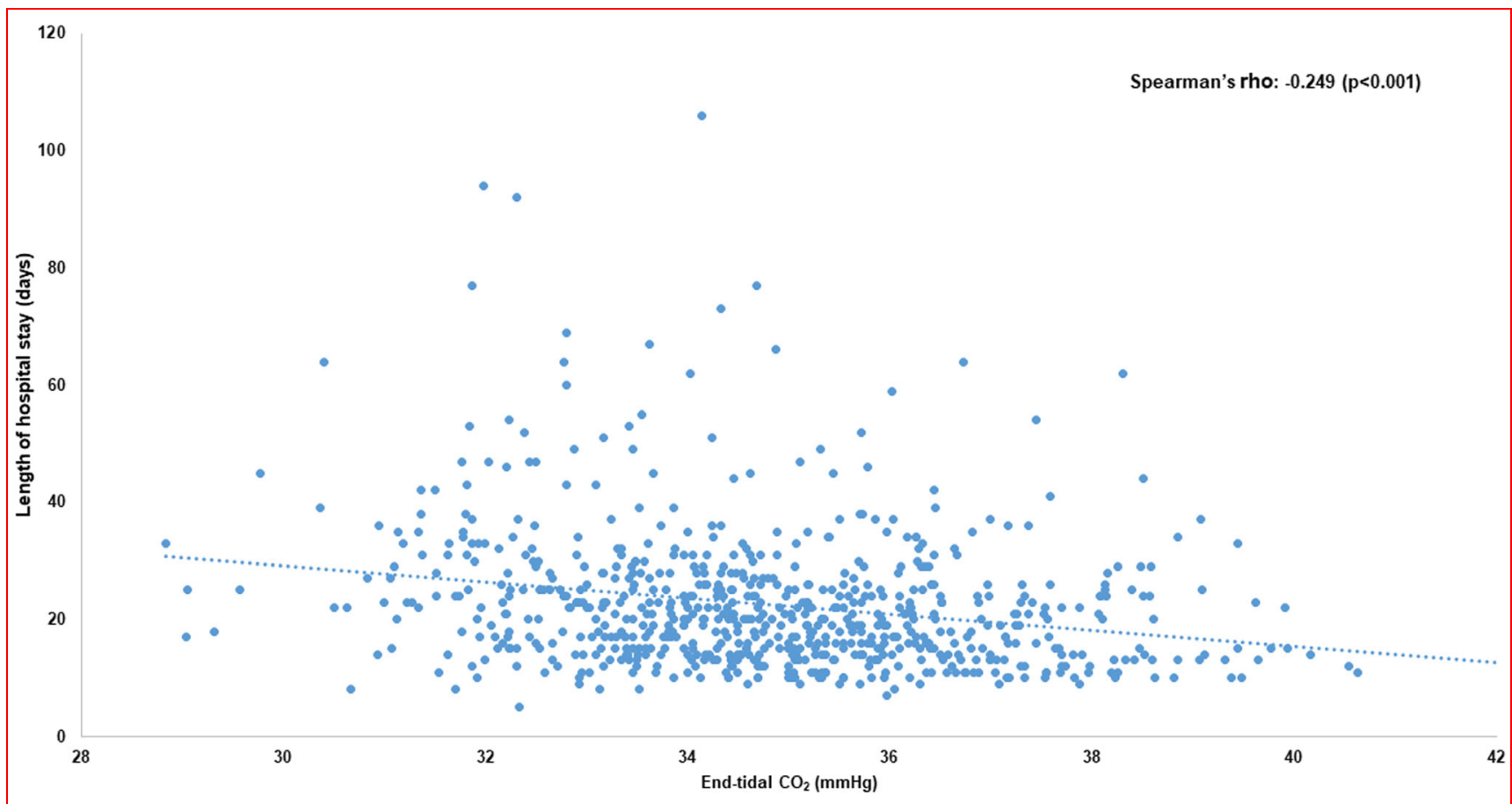

Fig. 3 Spearman's correlation analysis between end-tidal carbon dioxide $\left(\mathrm{EtCO}_{2}\right)$ and length of hospital stay (LOS)

show that hypocapnia has a correlation with negative impact on patient outcomes, and whether normocarbia or hypercapnia is more beneficial than normocapnia should be studied further.

In general, $\mathrm{EtCO}_{2}$ can be monitored simply and noninvasively, and it correlates with the arterial partial pressure of carbon dioxide $\left(\mathrm{PaCO}_{2}\right)$; therefore, $\mathrm{EtCO}_{2}$ is an essential parameter to be monitored during anesthesia. Two previous studies presented and analyzed only $\mathrm{EtCO}_{2}$ values; the present study added analysis of $\mathrm{PaCO}_{2}$ values and found that these values were also significantly different between the two groups. However, unlike the gradient between the two variables, which is known to be $2-5 \mathrm{mmHg}$, it was found that the $\mathrm{PaCO}_{2}-\mathrm{EtCO}_{2}$ differences were negative and the hypocapnic group was more hypocarbic, while the normocapnic group had less hypocarbia. Reversal of the gradient can be observed normally in pediatric, pregnant, and obese patients, caused by low functional residual capacity (FRC) and decreased total lung compliance, mechanical ventilation with large tidal volumes and low frequency, increased cardiac output and $\mathrm{CO}_{2}$ production [16-19]. FRC is inevitably reduced in patients undergoing upper abdominal surgery under general anesthesia [20]; therefore, the trend of negative $\mathrm{PaCO}_{2}-\mathrm{EtCO}_{2}$ in the present study is a probable clinical manifestation. However, $\mathrm{EtCO}_{2}$ is the average value over the entire operation period, while $\mathrm{PaCO}_{2}$ is the value obtained a few moments during the operation, particularly at the moment an anesthesiologist needs to be aware of a patient's blood gas or electrolyte status; therefore, it is not proper to predict that the negative trend of $\mathrm{PaCO}_{2}-\mathrm{EtCO}_{2}$ differences was maintained during the entire operation and in all patients. In accordance with previous studies, it can be concluded that $\mathrm{EtCO}_{2}$ value can predict of patients' outcomes, and this result is clinically practical because $\mathrm{EtCO}_{2}$ is continuously monitored in all anesthetized patients, whereas $\mathrm{PaCO}_{2}$ requires an invasive technique for determination and is not continuously monitored.

This study was a retrospective study, and it is likely that patient characteristics are not comparable between the two groups. Furthermore, no information was recorded regarding the strategies of the ventilator settings. The concept of lung protective ventilation was not popular about $10-15$ years ago; when comparing the annual patient distribution, the number of patients increased from hypocapnia to normocapnia over time. Anesthesiologists might have applied a familiar tidal volume and respiratory rate, rather than a cautious ventilator setting considering both height and weight. As a result, patients who were lightweight may have hyperventilated, resulting in a low $\mathrm{EtCO}_{2}$ in the hypocapnic group in the present study.

Dony and colleagues studied hospital stay as an indicator of healthcare quality as well as mortality as an indicator of the quality of critical care, and they found an increased 30-day mortality in hypocapnic patients [6]. In our study, mortality was increased for 30-day, 1-year, and 
Table 3 Perioperative factors affecting postoperative length of hospital stay

\begin{tabular}{|c|c|c|c|c|c|c|}
\hline \multirow[t]{2}{*}{ Variables } & \multicolumn{3}{|c|}{ Univariate } & \multicolumn{3}{|c|}{ Multiple } \\
\hline & HR & $95 \% \mathrm{CI}$ & $p$ value & HR & $95 \% \mathrm{CI}$ & $p$ value \\
\hline Hypocapnia & 1.52 & $1.32-1.75$ & $<.001$ & 1.61 & $1.37-1.89$ & $<0.001$ \\
\hline Female & 0.99 & $0.85-1.15$ & 0.893 & & & \\
\hline Age & 1.01 & $1.01-1.02$ & 0.010 & 1.02 & $1.01-1.03$ & $<0.001$ \\
\hline Operation time & 1.00 & $1.00-1.01$ & 0.585 & & & \\
\hline \multicolumn{7}{|l|}{ ASA classification } \\
\hline 2 & 0.84 & $0.70-1.02$ & 0.067 & 0.81 & $0.66-0.98$ & 0.028 \\
\hline 3 & 0.76 & $0.60-0.96$ & 0.022 & 0.83 & $0.63-1.09$ & 0.167 \\
\hline 4 & 0.52 & $0.21-1.27$ & 0.145 & 0.66 & $0.27-1.64$ & 0.367 \\
\hline \multicolumn{7}{|l|}{ Surgeon } \\
\hline 1 & 1.01 & $0.84-1.23$ & 0.896 & 0.82 & $0.67-1.01$ & 0.015 \\
\hline 2 & 2.36 & $1.90-2.92$ & $<0.001$ & 2.13 & $1.69-2.67$ & $<0.001$ \\
\hline 3 & 1.44 & $1.13-1.83$ & 0.009 & 1.21 & $0.93-1.58$ & 0.831 \\
\hline 4 & 1.31 & $0.94-1.82$ & 0.145 & 1.18 & $0.84-1.66$ & 0.789 \\
\hline Heart rate & 1.00 & $1.00-1.01$ & 0.749 & & & \\
\hline Systolic blood pressure & 1.00 & $0.99-1.01$ & 0.613 & & & \\
\hline Diastolic blood pressure & 1.00 & $0.99-1.01$ & 0.875 & & & \\
\hline Central venous pressure & 0.98 & $0.95-1.01$ & 0.111 & & & \\
\hline Peak airway pressure & 0.97 & $0.94-1.01$ & 0.060 & & & \\
\hline Hypertension & 0.95 & $0.83-1.11$ & 0.555 & & & \\
\hline Diabetes mellitus & 0.94 & $0.81-1.01$ & 0.493 & & & \\
\hline Pulmonary tuberculosis & 0.76 & $0.57-1.02$ & 0.067 & & & \\
\hline Hepatitis & 0.88 & $0.57-1.39$ & 0.597 & & & \\
\hline Asthma & 0.43 & $0.14-1.33$ & 0.141 & & & \\
\hline Stroke & 0.70 & $0.48-1.01$ & 0.054 & & & \\
\hline Heart disease & 0.78 & $0.50-1.20$ & 0.249 & & & \\
\hline POPF & 1.33 & $1.14-1.59$ & 0.001 & 1.41 & $1.18-1.69$ & $<.001$ \\
\hline
\end{tabular}

Values are presented as odds ratios (95\% confidence interval). $H R$ hazard ratio; $C I$ confidence interval; ASA American Society of Anesthesiologists; $P O P F$ postoperative pancreatic fistula

2-year periods, but there were no statistically significant differences between the normocapnic and hypocapnic groups. First, the 30-day mortality of PPPD was less than $1 \%$, which makes it difficult to demonstrate statistical significance in a few hundred patients. Regarding the 1and 2-year mortality, they might be affected by disease progression or severity, such as cancer stage and recurrence, rather than by acute postoperative recovery. Alternatively, more patients may be needed to reveal the effect of hypocapnia on this long-term outcome.

POPF remains the main source of major morbidity and mortality after PPPD, which can occur in $13-41 \%$ of postoperative patients [21, 22]. Patients who develop POPF are also more likely to develop postoperative complications, including prolonged LOS, wound infection, bile leak, and acute cardiac events [23]. POPF was a significant factor that also led to prolonged LOS in this study
(Table 3); even so, $\mathrm{EtCO}_{2}$ did not seem to be related to POPF.

This study has several limitations. First, this is a retrospective study that reveals the relationship between phenomena and outcome, and the factors affecting LOS are so complex and diverse that it is difficult to investigate the exact mechanism of $\mathrm{EtCO}_{2}$ on LOS. Further prospective investigations are warranted to determine if there is a causal relationship. Second, because of the technical limitations of our electronic medical record system, ICU stays were measured on a daily basis rather than on an hourly basis; this may have affected the capacity to discern differences. Third, there was no analysis regarding the influence of other complications on LOS, as the incidence of complications besides POPF was minimal $(<1 \%)$ and less than expected. This may be due to insufficient medical records. The development of POPF, the major 
complication of PPPD, was well-documented in the medical records and was thus able to be analyzed in this study. Fourth, four surgeons were involved in the study over 10 years. Even though their surgical skills and outcomes were considered comparable and their discharge criteria were standardized, it is difficult to assume that they were perfectly identical. Finally, the LOS of PPPD can differ depending on each country's medical system including insurance, or each institution's policy or the application of the enhanced recovery after surgery (ERAS) protocol. Therefore, there is an issue of external validity. Further studies are needed on the effects of EtCO2 in institutions with shorter hospital stays.

In summary, intraoperative end-tidal $\mathrm{CO}_{2}$ status significantly influenced LOS in our cohort of patients undergoing PPPD. This study suggests that preventing hypocapnia during general anesthesia may shorten the length of hospital stay after major abdominal surgery such as PPPD. A prospective study is warranted to demonstrate causality and to determine the best $\mathrm{CO}_{2}$ management strategy for these and other procedures.

Acknowledgement This research received no external funding.

\section{Compliance with ethical standards}

Conflicts of interest All authors declare no conflicts of interest.

Ethical approval This study was approved by the local ethics committee of the hospital, and the need for informed consent was waived.

\section{References}

1. Huttner FJ, Fitzmaurice C, Schwarzer G, et al. (2016) Pyloruspreserving pancreaticoduodenectomy ( $\mathrm{pp}$ Whipple) versus pancreaticoduodenectomy (classic Whipple) for surgical treatment of periampullary and pancreatic carcinoma. Cochrane Database Syst Rev 2:Cd006053

2. Akkermans A, van Waes JAR, Thompson A et al (2019) An observational study of end-tidal carbon dioxide trends in general anesthesia. Can J Anaesth 66:149-160

3. Curley G, Kavanagh BP, Laffey JG (2010) Hypocapnia and the injured brain: more harm than benefit. Crit Care Med 38:1348-1359

4. Laffey JG, Kavanagh BP (2002) Hypocapnia. N Engl J Med 347:43-53

5. Whitesell R, Asiddao C, Gollman D et al (1981) Relationship between arterial and peak expired carbon dioxide pressure during anesthesia and factors influencing the difference. Anesth Analg 60:508-512

6. Dony P, Dramaix M, Boogaerts JG (2017) Hypocapnia measured by end-tidal carbon dioxide tension during anesthesia is associated with increased 30-day mortality rate. J Clin Anesth 36:123-126

7. Wax DB, Lin HM, Hossain S et al (2010) Intraoperative carbon dioxide management and outcomes. Eur J Anaesthesiol $27: 819-823$

8. Way M, Hill GE (2011) Intraoperative end-tidal carbon dioxide concentrations: what is the target? Anesthesiol Res Pract 2011:271539

9. Bassi C, Dervenis C, Butturini G et al (2005) Postoperative pancreatic fistula: an international study group (ISGPF) definition. Surgery 138:8-13

10. Gittell JH, Fairfield KM, Bierbaum B et al (2000) Impact of relational coordination on quality of care, postoperative pain and functioning, and length of stay: a nine-hospital study of surgical patients. Med Care 38:807-819

11. Saghaei M, Matin G, Golparvar M (2014) Effects of intra-operative end-tidal carbon dioxide levels on the rates of post-operative complications in adults undergoing general anesthesia for percutaneous nephrolithotomy: a clinical trial. Adv Biomed Res 3:84

12. Mutch WAC, El-Gabalawy R, Girling L et al (2018) End-tidal hypocapnia under anesthesia predicts postoperative delirium. Front Neurol 9:678

13. Fleischmann E, Herbst F, Kugener A et al (2006) Mild hypercapnia increases subcutaneous and colonic oxygen tension in patients given $80 \%$ inspired oxygen during abdominal surgery. Anesthesiology 104:944-949

14. Helmerhorst HJ, Roos-Blom MJ, van Westerloo DJ et al (2015) Associations of arterial carbon dioxide and arterial oxygen concentrations with hospital mortality after resuscitation from cardiac arrest. Crit Care 19:348

15. Hovorka J (1982) Carbon dioxide homeostasis and recovery after general anaesthesia. Acta Anaesthesiol Scand 26:498-504

16. Fletcher R, Jonson B (1984) Deadspace and the single breath test for carbon dioxide during anaesthesia and artificial ventilation. Effects of tidal volume and frequency of respiration. Br J Anaesth $56: 109-119$

17. Shankar KB, Moseley H, Kumar Y (1991) Negative arterial to end-tidal gradients. Can J Anaesth 38:260-261

18. Shankar KB, Moseley H, Kumar Y et al (1986) Arterial to end tidal carbon dioxide tension difference during caesarean section anaesthesia. Anaesthesia 41:698-702

19. Rich GF, Sconzo JM (1991) Continuous end-tidal $\mathrm{CO}_{2}$ sampling within the proximal endotracheal tube estimates arterial $\mathrm{CO}_{2}$ tension in infants. Can J Anaesth 38:201-203

20. Spadaro S, Karbing DS, Mauri T et al (2016) Effect of positive end-expiratory pressure on pulmonary shunt and dynamic compliance during abdominal surgery. Br J Anaesth 116:855-861

21. Berger AC, Howard TJ, Kennedy EP, et al (2009) Does type of pancreaticojejunostomy after pancreaticoduodenectomy decrease rate of pancreatic fistula? A randomized, prospective, dual-institution trial. J Am Coll Surg 208:738-747; discussion 747-739

22. Iacono C, Verlato G, Ruzzenente A et al (2013) Systematic review of central pancreatectomy and meta-analysis of central versus distal pancreatectomy. Br J Surg 100:873-885

23. Raty S, Sand J, Lantto E et al (2006) Postoperative acute pancreatitis as a major determinant of postoperative delayed gastric emptying after pancreaticoduodenectomy. J Gastrointest Surg 10:1131-1139

Publisher's Note Springer Nature remains neutral with regard to jurisdictional claims in published maps and institutional affiliations. 